\title{
Pilot randomised controlled trial of a brief mindfulness-based intervention for those with persistent pain
}

\author{
Ana Howarth ${ }^{1}$ (1) Muhammad Riaz $^{1} \cdot$ Linda Perkins-Porras $^{2} \cdot$ Jared G. Smith $^{1}$ • \\ Jeevakan Subramaniam ${ }^{1}$ - Claire Copland ${ }^{3} \cdot$ Mike Hurley $^{4} \cdot$ Iain Beith $^{4}$. \\ Michael Ussher ${ }^{1,5}$
}

Received: August 9, 2018/Accepted: April 4, 2019/Published online: April 22, 2019

(C) The Author(s) 2019

\begin{abstract}
A pilot-randomised controlled trial (RCT) examined the effects of a brief mindfulness-based intervention (MBI) on persistent pain patients and assessed the feasibility of conducting a definitive RCT. A brief (15 min) mindfulness body-scan audio was compared with an active control administered in a clinic and then used independently over 1 month. Immediate effects of the intervention were assessed with brief measures of pain severity, distraction and distress. Assessments at baseline, 1 week and 1 month included pain severity and interference, mood, paincatastrophizing, mindfulness, self-efficacy, quality of life and intervention acceptability. Of 220 referred patients, 147 were randomised and 71 completed all assessments. There were no significant immediate intervention effects. There were significant positive effects for ratings of intervention 'usefulness' at 1 week $(p=0.044)$, and pain self-efficacy at 1 month $(p=0.039)$ for the MBI group compared with control. Evidently, it is feasible to recruit persistent pain patients to a brief MBI study. Strategies are needed to maximise retention of participants.
\end{abstract}

Ana Howarth

p1306542@sgul.ac.uk

1 Population Health Research Institute, St George's, University of London, Cranmer Terrace, London SW17 0RE, UK

2 Institute of Medical and Biomedical Education, St George's, University of London, London, UK

3 Persistent Pain Service, St George's Hospital, London, UK

4 Faculty of Health, Social Care and Education, Kingston University and St George's, University of London, London, UK

5 Institute for Social Marketing, University of Stirling, Stirling FK9 4LJ, UK
Trial registration Current controlled trials ISRCTN6 1538090. Registered 20 April 2015.

Keywords Persistent pain - Mindfulness - Intervention · Randomised controlled trial · Pilot

\section{Introduction}

Persistent pain (i.e., chronic pain) is a major health issue that impacts people regardless of socioeconomic status, gender or access to healthcare (Rice et al., 2016). Within the United Kingdom alone, between one-third and one-half of the population are affected by persistent pain (Fayaz et al., 2016). It has a negative impact on quality of life (Bridges, 2012) and results in high levels of disability (Fredheim et al., 2008) with $41 \%$ of patients attending pain clinics reporting being unable to work (British Pain Society, 2012). Furthermore, high comorbidity rates of depression and anxiety (Elliott et al., 2003) are common and 16\% of sufferers report their persistent pain is so bad that they sometimes want to die (Sir Liam Donaldson, 2008).

Psychological therapies, most commonly in the form of cognitive behavioural therapies (Eccleston et al., 2009; Morley et al., 1999) have been shown to play an important role in helping patients cope with persistent pain (Roditi \& Robinson, 2011; Williams et al., 2012). More recently mindfulness-based approaches have emerged (Hayes, 2004; Harrison et al., 2017). These interventions typically involve training patients to engage in self-regulation of attention through increasing awareness of, and accepting, present thoughts, feelings and physical sensations (KabatZinn, 1990). The translation of mindfulness-based practices into a secular health care intervention was initiated by Kabat-Zinn in the 1970s when he investigated persistent 
pain management at the University of Massachusetts medical school (Kabat-Zinn, 1982). During this time, patients were trained in mindfulness and the result was the development of a 10 week structured program called Mindfulness-based Stress Reduction (MBSR) (Kabat-Zinn et al., 1985), which was later reduced to what is now the traditional 8 week program.

Since then, good evidence for full length mindfulnessbased interventions (MBIs) in both clinical and non-clinical populations has been established (Bawa et al., 2015; Goyal et al., 2014; Hilton et al., 2017). Among those with persistent pain, MBIs have been shown to reduce anxiety, depression and distress, and to enhance quality of life (Hofmann et al., 2010) while at the same reducing negative habitual responding which positively impacts pain distress and exacerbation (Grossman et al., 2004; Kabat-Zinn, 1990). There is also evidence that regular mindfulness meditation modulates neural mechanisms (Zeidan et al., 2011, 2012), especially those related to pain, as well as benefitting inflammatory systems (Greeson, 2008). In addition, recent UK National Health Service (NHS) guidelines include a recommendation for mindfulness meditation in treating depression (NCCMH, 2009).

While this research is promising, a major barrier with the implementation of current MBIs is the amount of time they require and the necessity of a trained specialist to oversee them (WHO, 2003). Mindfulness programmes are typically administered over 8 weeks and involve group sessions. Many persistent pain patients do not have the resources, physically or mentally, to engage with such an intensive programme (BPS, 2008; Sim \& Lewis, 2012). Self-help type interventions, which offer more autonomy, are likely to be more adaptable for many such patients and the self-management model of care is now an integral part of the NHS (Rogers \& Kennedy, 2008). One type of brief intervention that fits this profile is a short mindfulnessbased body scan. This scan is a key component of mindfulness meditation practice; it involves being directed to focus attention on the present moment through observing the breath and bodily sensations, while becoming aware of, and accepting without judgement, any thoughts and feelings which arise. The traditional mindfulness-based stress reduction (MBSR) intervention includes a body scan (Baer, 2003), usually lasting $45 \mathrm{~min}$, although sometimes shortened.

Investigations with healthy populations, with a brief MBI, have been successful in demonstrating a reduction in some aspects of the pain experience, such as distress and sensitivity, during experimental pain studies (Liu et al., 2013; Zeidan et al., 2010a). However, in other studies (Prins et al., 2014; Sharpe et al., 2013) where experimental pain was applied to healthy participants, there were no significant results. In a persistent pain population, encour- aging effects were found, with an audio recording of a 10 min body scan reducing reports of distress, immediately after listening to the audio, in a clinical setting (Ussher et al., 2012). This same study also found no effects when repeated in the participants' own environment. To further explore what appeared to be a promising intervention within a clinical population, a qualitative study (Howarth et al., 2016a, b) was conducted which informed the current study in relation to key refinements of the previously used intervention mostly by extending the duration (i.e., use for 1 week requested and up to 1 month, encouraged) and length (i.e., $15 \mathrm{~min}$ instead of $10 \mathrm{~min}$ ). As well, as the nature of the intervention was modified to be more selfmanagement focused, a selection of different outcome measures that were considered more relevant were piloted. The aim of the current study was to evaluate the effects of a brief MBI, which is a refinement of the intervention used in the latter study and assess the feasibility of conducting a definitive randomised controlled trial (RCT).

\section{Methods}

\section{Design}

This was a single centre, parallel group, RCT pilot study, designed to assess the immediate effects of a MBI, as well as the feasibility of conducting a definitive RCT. According to Bowen et al.'s (2009) article on design feasibility studies, this study could be considered an acceptability, demand, implementation and practicality feasibility study based on the nature of the questions the study is asking and the variety of outcomes of interest (Bowen et al., 2009).

Ethical approval was given by the NRES Committee London-Camden \& Islington (14/LO/1912). Participants provided written informed consent.

\section{Participants}

Patients were recruited from three outpatient NHS physiotherapy and pain clinics at in south London. All patients were initially screened by a clinician (i.e., physiotherapist or pain consultant). Those who met the inclusion criteria were given a patient information sheet (PIS) by the clinician and were asked if they consent to have their contact details passed to a researcher, who would then call to discuss whether they wished to join the study. Or if they preferred they could meet with the researcher in person to discuss the study.

Patients were eligible if they were over 18 years of age, living with persistent pain [i.e., with a diagnosis of persistent pain or having had pain for more than 3 months past the time healing should have occurred (BPS, 2008)], and 
able to hear audio recordings or have equipment to enable them to do so. The clinicians were asked to whether they thought the intervention would be too burdensome for their patient's health and wellbeing. Patients were excluded if they were considered too unwell to participate by the clinician or were unable to speak or read English sufficiently to understand and complete the self-administered questionnaires.

\section{Sample size}

It is recommended that pilot/feasibility studies ideally recruit a total of at least 50 participants (Sim \& Lewis, 2012). We aimed to recruit 90 participants (45 in each treatment arm). Then allowing for 10 participants withdrawing [estimate based on a previous mindfulness study with a similar population (Ussher et al., 2012)] we aimed to have approximately 80 participants with data through to the final 1 month follow-up. Moreover, for the immediate effects of the intervention, based on previous findings (Ussher et al., 2012), we estimated that a sample size of 25 in each of the two groups (total sample $\mathrm{N}=50$ ) would have $80 \%$ power to detect an effect size (Cohen's d) of 0.6 with a 5\% two-sided significance level, when comparing scores on the perceived distress scale after the intervention. We chose the distress measure on which to base the latter power calculation as this was the only key outcome measure for which we had data from similar previous studies.

\section{Randomisation}

An independent statistician (MR) generated a randomization list using the online resource 'Research Randomizer' (Urbaniak \& Plous, 2013) who was then blinded to group allocation. This list was used by researchers to allocate volunteers to either the control or MBI group on a 1:1 basis. Patients were allocated their number in ascending order based on order of enrolment. Allocation was concealed from the participant and researcher until all baseline assessments were completed. Due to limited resources, the same researcher delivered the intervention and administered the research measures and neither participants nor researchers were blinded to treatment allocation during intervention delivery or during outcome assessment. An independent researcher (MU), who was blinded to the treatment allocation, conducted the initial analysis for the main outcomes.

\section{Interventions}

To improve the reporting of the interventions, the Template for Intervention Description and Replication (TIDieR) (Hoffmann et al., 2014) and SPIRIT (Standard Protocol
Items: Recommendations for Interventional Trials) (Chan et al., 2013) checklists were used to guide the description of the interventions.

\section{Mindfulness-based intervention group: Brief self- management mindfulness-based audios}

Patients in the MBI group were given an audio recording of a 15 min mindfulness body scan on an MP3 player (with earphones) or were offered the option of having the audio downloaded directly to a personal device of their choice, such as a smart phone or iPad.

The choice of the body scan meditation for the audio was based on successful traditional MBSR interventions, which routinely include a body scan meditation as the introductory exercise. In comparison to other mindfulness exercises, such as breathing or walking meditations, this particular exercise is considered to be an accessible introduction to mindfulness meditation (Kabat-Zinn, 1990). In a clinical setting with a persistent pain population, a brief (10 min) body scan was found to reduce reports of distress, immediately after listening to the audio (Ussher et al., 2012).

The body scan used in this study was an extended version of a 10 min body scan that was used in a previous qualitative study (Howarth et al., 2016a, b) investigating the acceptability of the intervention to patients. It is based on a transcript from Breathworks (Breathworks, 2013), an established mindfulness organization specialising in supporting those with persistent pain. As part of the prior qualitative study (31), and in response to feedback from patients, the intervention was extended from 10 to $15 \mathrm{~min}$ so that it would feel less rushed.

The audio recording directed the listener to 'scan' their body with their attention systematically, starting with the toes and finishing with the crown of the head. Throughout this process, the listener was also encouraged to be aware of their breathing and to accept all thoughts and feelings, whether positive or negative, without trying to alter them in any way. The audio was administered in the presence of a researcher in the first instance, in a clinical setting (i.e., physiotherapy or pain clinic medical side room or cubicle) and a telephone follow up at 1 week and 1 month was conducted (in nearly all cases) by the same researcher. Use of the audio in the patient's own environment at least three further times during the first week was requested and after that use was encouraged but no set number of times was prescribed for the subsequent 3 weeks as the main aim was to see if patients would choose to continue to use the audio of their own volition. Following administration of the MBI, a study packet including information and instructions for use of the audios along with brief information regarding mindfulness (i.e., frequently asked questions) and ques- 
tionnaires to be filled out at home, were given to the patient. The inclusion of an information sheet was developed in response to patient feedback in the previous qualitative study (Howarth et al., 2016a, b).

In order to offer some variety, an audio of a mindfulness breathing meditation and a mindfulness moving meditation were given (i.e., loaded onto the MP3 player or device) to the MBI group as well, but use was not recommended until after 1 week. The breathing meditation was an exercise where the breath is used as an object of concentration and the listener is asked to focus on the sensations of breathing (e.g., the feeling of the chest rising and falling). The moving meditation was focused on gentle exercises (e.g., small wrist twists or arm movements), which could be done sitting or standing and the listener was guided to pay attention to bodily sensations after making each movement. This variety in mediation was partly to match the variety that the control group would be experiencing as they would not be listening to the same content regularly (i.e., a different chapter each session) but also to echo the structure of traditional MBI's which offer more mindfulness exercises on a weekly basis so as to motivate and encourage growth of the practice. Both of the additional meditations were also based on transcripts from Breathworks.

\section{The control group: distraction audios}

Patients in the control group were given eight, $15 \mathrm{~min}$ audio recordings of sequential readings from "The English Village: History and Traditions" (Wainwright, 2011), which is a non-fiction book considered not to include any strong emotive content. The readings started from the beginning of the book and it was hoped that enough interest would be generated as the story progressed to encourage patients to listen to a total of three further sessions in the first week. In total, eight sessions were recorded with the intention that four recordings would be used in the first week and that the remaining four could be used in the following 3 weeks. As with the MBI group, patients were given an MP3 player (with earphones) or the option of having the audios downloaded directly to a personal device. For the first session in clinic, the first of these sequential readings, which was also the first section of the book, was presented. Non-fiction material, similar in style and content, has been used in a previous study examining the acute effects of mindfulness among those with persistent pain, where it was found to be an acceptable intervention (Ussher et al., 2012). Recordings were made using the same narrator as the intervention, and were read at a similar pace and with comparable pauses.

As with the MBI group, use of the audios was requested at least three further times during the first week. After that, continuing use was encouraged, with no set prescription for the subsequent 3 weeks. Following administration of the control intervention the study packet including information and instructions for use of the audios (minus the mindfulness frequently asked questions that were included for the MBI group) and questionnaires to be filled out at home, were given to the patient.

\section{Procedure in clinic}

Patients who met the inclusion criteria were approached by the research team and given the PIS. Patients were given as much time as they needed to consider whether they wanted to participate. To standardise delivery a researcher checklist was followed and the three researchers observed each other administering the intervention to at least one patient each.

As the intervention was intended to be a self-management tool, only the initial session was conducted in clinic, face-to-face with a researcher in a private room or cubicle. Patients were asked to complete baseline measures, randomised to either the control or MBI group, asked to complete brief psychological measures, and then to listen to the relevant audio once in clinic with the researcher. Immediately after listening to the audio, patients were asked to complete the brief psychological measures again. Before leaving, patients were advised to consider barriers and facilitators to use of the audio in their own environment and were given a study packet to take home. They were instructed to use the audios as a self-management tool a minimum of three times within the first week and to try the audio during particularly painful times if possible. With a lack of previous evidence offering guidance for the usage amount within a clinical population, two sources were combined to inform the recommendation for this study. Brief MBIs in experimental studies with non-clinical populations tended to average between 3 and 4 times weekly. This recommendation was combined with consultation with an expert in the area of chronic pain treatment (i.e., a clinical pain psychologist).

The contents of the study packet containing follow-up questionnaires, (i.e., study diaries $1,2, \& 3$, detailed below with measures), self-addressed prepaid return envelopes and brief instructions, were then reviewed with the patient in case there were queries. If the audios were not directly downloaded to a personal device, patients were invited to keep the MP3 players. The offer of the MP3 player was not mentioned in the PIS and therefore was not considered as an incentive to recruitment. 


\section{Measures and schedule of assessment}

\section{Baseline data collection}

Patients were asked to provide demographic details including age, marital status, occupation, education, and ethnic group along with five pain related questions, namely: "What is your clinical diagnosis?", "How long have you been living with your pain?", "Are you currently taking any medication for your pain and if so, which one/ s?", "Over the last week, how confident have you been in managing your pain" ( $1=$ not at all confident to $7=\mathrm{ex}-$ tremely confident, i.e., pain self-efficacy) and "During the past week, how much has your work or other regular daily activities been limited as a result of your pain symptoms?" ( $1=$ not at all to $5=$ extremely). They then completed a measure of mood [Hospital Anxiety and Depression Scale (Zigmond \& Snaith, 1983)], a mindfulness questionnaire [Cognitive and Affective Mindfulness Scale-Revised (Feldman et al., 2007)], a pain specific questionnaire [Brief Pain Inventory (Cleeland \& Ryan, 1994)], a pain catastrophizing questionnaire [Pain Catastrophizing Scale (Sullivan et al., 1995)] and a health related quality of life (HRQoL) questionnaire [EQ-5D-5L (Herdman et al., 2011)]. Immediately before and after the initial use of the audio in clinic, patients were asked to complete three questions regarding their level of distraction, pain severity and pain distress $(1=$ not at all to $5=$ extremely $)$. Full details of the measures are given below.

\section{Measures completed during the first week}

Study Diary 1 included a self-monitoring table detailing date, time and position of use (e.g., sitting or lying) of the audios and a repeat of the baseline brief measures of level of distraction, pain severity and pain distress immediately before and after the last session of listening to the audio during the first week.

\section{Measures completed after 1 week}

Study Diary 2 included a brief questionnaire where patients are asked: "How useful did you find the audio guide for helping you to relax?" ( $1=$ not at all to $5=$ extremely useful), and "Would you recommend this audio guide to others to help manage their persistent pain?" $(1=$ definitely would not recommend to $5=$ definitely would recommend it). To assess level of experience of activities related to mindfulness, the question: "Have you had experience of yoga, tai-chi or any type of meditation?" ( $1=$ no experience of these activities to $7=\mathrm{I}$ currently practice these activities at least once a week) was included.
These questions were followed by a repeat of the measure of mindfulness that was completed at baseline.

\section{Measures completed during and after 1 month}

Study Diary 3 included another self-monitoring table where patients could continue to detail date, time and position of use of the audios during the 3 weeks prior to the final 1 month follow up. At 1 month, items regarding pain self-efficacy and physical function were repeated in addition to the measures of mood, pain catastrophising, mindfulness, and HRQoL that were administered at baseline. It was considered that 1 week was likely too soon for patients to make detectable changes in physical and/or psychological function, therefore these measures were only administered after the completion of the intervention at 1 month.

A brief assessment of whether participants had continued listening to the audio (and if so, how often), a discussion of the main barriers to and facilitators of use, and views on options such as an online support group forum, texting support and more face time, was conducted with a brief (approximately $5 \mathrm{~min}$ ) open-ended telephone interview. A schedule of assessment for all measures included is presented in Table 1 below.

\section{Intervention behaviour change techniques at 1 week}

Behaviour change techniques were included to maximise engagement and adherence. At 1 week, the researcher followed up by telephone and encouraged continued use of the intervention, identified perceived barriers to and facilitators of use and set goals with the patient by recommending continued use of the intervention at least three times a week. Self-monitoring by diary was encouraged also. These behaviour change techniques (BCTs) come under the labels "Goal setting" or "Action planning", "Self-monitoring of behaviour" and "Problem solving" as per the generic BCT Taxonomy (v1) (Michie et al., 2013).

\section{Debrief at 1 month}

Patients were followed up after 1 month by telephone and were debriefed regarding the full nature of the study, and if they were part of the control group, they were offered to have the MBI audios sent to them. Resources that were readily available to the public were recommended at this time if patients wished to further explore mindfulness. Patients were reminded to post back the questionnaires. 
Table 1 Schedule of data and measurement collection

\begin{tabular}{|c|c|c|c|c|c|}
\hline Measure & Baseline & During week & At 1 week & During month & At 1 month \\
\hline Background and pain related questionnaire & $X$ & & & & \\
\hline Pain self-efficacy item & $X$ & & & & $\mathrm{X}$ \\
\hline Pain and physical function item & $X$ & & & & $\mathrm{X}$ \\
\hline Mood questionnaire $\left(\mathrm{HADS}^{\mathrm{a}}\right)$ & $\mathrm{X}$ & & & & $\mathrm{X}$ \\
\hline Mindfulness questionnaire $\left(C A M S-R^{a}\right)$ & $\mathrm{X}$ & & $\mathrm{X}$ & & $\mathrm{X}$ \\
\hline Pain specific questionnaire $\left(\mathrm{BPI}^{\mathrm{a}}\right)$ & $\mathrm{X}$ & & & & \\
\hline Pain catastrophizing questionnaire $\left(\mathrm{PCS}^{\mathrm{a}}\right)$ & $\mathrm{X}$ & & & & $X$ \\
\hline HRQoL questionnaire (EQ-5D-5L ${ }^{\mathrm{a}}$ ) & $X$ & & & & $X$ \\
\hline Brief psychological measures (two times, before and then after intervention) & $\mathrm{X}$ & $\mathrm{X}$ & & & \\
\hline Experience of audio items (i.e. usefulness for relaxing) & & & $X$ & & \\
\hline Previous experience & & & $\mathrm{X}$ & & \\
\hline Self-monitoring table & & $X$ & & $\mathrm{X}$ & \\
\hline
\end{tabular}

${ }^{a} H A D S$ hospital anxiety and depression scale, $C A M S-R$ cognitive and affective mindfulness scale revised, $B P I$ brief pain inventory, $P C S$ pain catastrophizing scale, $E Q-5 D-5 L$ EuroQol-5 dimensions-5 levels

\section{Measures}

\section{Hospital anxiety and depression scale}

The Hospital Anxiety and Depression Scale (HADS) designed by Zigmond \& Snaith (1983) (Zigmond \& Snaith, 1983) has been widely used as a tool to assess the severity of depression and anxiety and is an easily-administered screening questionnaire. It includes fourteen items, seven measuring anxiety and seven measuring depression. The respondent must choose one of four responses for each item in accordance with how they have felt over the previous week. A score of 0-21 is calculated for each disorder with total scores between 11-21 indicating abnormal levels of anxiety or depression (Crawford et al., 2001). The HADS has been routinely used for research within chronic pain populations (Kalia \& O'Connor, 2005; Sagheer et al., 2013; Tang et al., 2007; Veehof et al., 2011) and has been found to have good internal consistency for both the anxiety $(\mathrm{a}=0.83)$ and the depression $(\mathrm{a}=0.84)$ subscales (Pallant \& Bailey, 2005).

\section{Cognitive and affective mindfulness scale-revised (CAMS-R)}

The Cognitive and Affective Mindfulness Scale-Revised (CAMS-R) (Feldman et al., 2007) is a 10-item scale which uses everyday language appropriate for those with little meditation experience. It is the revised version of the Cognitive and Affective Mindfulness Scale (CAMS) (Greg Feldman \& Hayes, 2005) which is an 18-item measure designed to capture mindfulness as a general daily experience. The CAMS-R has been compared with two other existing mindfulness measures, the Mindfulness Attention
Awareness Scale (Brown \& Ryan, 2003) and The Freiburg Mindfulness Inventory (Walach et al., 2006) where it was found to be positively correlated (MAAS $(r=0.51$, $p<0.001$, FMI $(\mathrm{r}=0.66, p<0.001)$ (Baer et al., 2006; Thompson \& Waltz, 2007) with an acceptable internal consistency $(\mathrm{a}=0.76)($ Feldman et al., 2007) which was a weakness of the original scale. The CAMS-R is also uniquely appropriate in that includes a measure related to psychological distress, which is highly relevant to the current study and chronic pain population.

\section{EuroQuol—5 dimensions—5 levels}

The EuroQol-5 Dimension-5 Levels (EQ-5D-5L) (Herdman et al., 2011) is the most recently developed version of the EQ-5 Dimensions (EQ-5D) (Brooks, 1996; EuroQol Group, 1990) that has good construct validity and responsiveness among people with chronic pain (Obradovic et al., 2013) and is a standardised measure of health status. It was developed by the Euroqol group, is supported by the National Institute for Clinical Excellence (NICE) for measuring change in health related quality of life with various patient groups (Brazier \& Longworth, 2011) and has been validated within numerous patient groups including the chronic pain population. It has been shown to be a sensitive tool with internal consistency $(a=0.78)$ (Cheung et al., 2016) and reliability (Dorman et al., 1997; Hurst et al., 1994; Marra et al., 2005; Mustur et al., 2009).

\section{The brief pain inventory}

The Brief Pain Inventory (BPI) (Cleeland \& Ryan, 1994) is a tool for the assessment of pain in both clinical and research settings, is easy to use and includes simple 
numeric rating scales from 0 to 10 (with $0=$ no pain to $10=$ pain as bad as you can imagine). The BPI has been used internationally (Cleeland \& Ryan, 1994; Gjeilo et al., 2007; Song et al., 2016) to measure severity and interference of pain in patients who live with a range of chronic pain presentations and has good internal consistency ranging from 0.80 to 0.87 for the severity items and 0.89 to 0.92 for the interference items (Cleeland \& Ryan, 1994).

\section{Pain catastrophizing scale}

The Pain Catastrophizing Scale (PCS) (Sullivan et al., 1995) is a 13-item scale consisting of statements in relation to the thoughts and feelings patients report when they experience pain. Scored from zero (not at all) to four (all the time), the total PCS scores range from 0 to 52 points and higher scores indicating higher levels of pain catastrophizing. The PCS was originally an elaboration on the Coping strategies Questionnaire (CSQ) (Rosenstiel \& Keefe, 1983) and now consists of three subscales, which are magnification, rumination, and helplessness. The scale was developed to be used within both clinical and nonclinical populations and has been shown to have reliability and validity in both pain populations and healthy adult populations with a high internal consistency $(a=0.87)$ (Osman et al., 2000).

\section{Brief measures completed before and after audio in clinic and the last session during the first week at home}

Three brief, single-item measures were used to assess level of distraction, pain severity and pain distress. Patients were asked to rate "Right now, I could be easily distracted.", "How severe are your pain related symptoms right now?", and "How distressing are your pain related symptoms right now?", all on a scale from 1 (not at all) to 7 (extremely so). The two pain-related items were based on a previous study (Ussher et al., 2012) with a similar intervention and the distraction item was developed specifically for the study, based on an item from the CAMS-R (i.e., "I am easily distracted.”).

\section{Statistical analysis}

We compared baseline characteristics for the two study groups (i.e., MBI and control), using t-tests, Mann-Whitney tests or Chi squared depending on the data. Baseline characteristics of non-completers (i.e., those randomised who did not complete the 1 month follow-up measures) were compared with the sample that did complete all follow-up measures.
For the analysis of the primary outcomes, which were the immediate effects of the intervention, we assessed the effect of the body scan intervention versus the control intervention on ratings for the brief psychological measures administered immediately before and after the interventions. This analysis was conducted with ratings made in the clinic and also for those conducted in the participant's own environment. It was hypothesized that patients in the brief MBI group would report reductions in ratings of distraction, pain severity and pain distress compared with the control condition. First, we conducted multiple linear regressions with the post intervention immediate effect scores as the dependent variables and treatment groups and baseline immediate effect scores as the independent variable (Vickers et al., 2018). Statistical significance was assessed using likelihood-ratio test, and the regression coefficient $(\beta)$ was reported as the estimate of effect given as mean difference of change scores with $95 \%$ confidence interval (CI). The effect estimates were adjusted for age, gender and baseline BPI score in the multiple regression analysis, as being potentially important prognostic baseline factors.

Next, we assessed the effect of the study groups on changes in outcome scores between baseline and 1 month for the HADS, EQ-5D-5L, PCS, CAMS-R, and ratings of "confidence in managing pain" and "limitations of ADL". Also we examined changes in the CAMS-R at 1 week. The study was not powered to detect significant differences between the groups and we carried out analyses to inform parameters for a definitive trial. We computed change scores between baseline and 1 month or 1 week and conducted multiple regressions, with adjustments as above.

To assess the impact of missing data on results, sensitivity analyses were conducted using multiple imputation for missing observations in any outcome variables. The imputation uses regression models to predict and impute values for missing observations, with the assumption that missing data (i.e., brief psychological outcome measures) are missing at random (MAR). Missing values in the rating scores for other measures at 1 week and 1 month were replaced by imputed values using chained equations (Azur et al., 2011; Van Buuren et al., 1999) (linear regression models) with the PMM method (Little, 1988; Morris et al., 2014; Rubin, 1986). The models for imputation were fitted with rating scores for the outcomes of immediate effects and other outcomes measures at follow ups as dependent variables and the rating scores at baseline, and the baseline characteristics of the patients as independent variables. In the linear regression model for the outcomes scores at 1 month's follow-up, the outcome scores at 1 week were also used as an explanatory variable. Twenty imputed datasets were created and the same analysis as described above for assessing the effect of the intervention on outcome scores, 
was repeated in these 20 datasets. The imputation-specific estimates for the effect of the intervention on the outcomes scores were combined using Rubin's rules (Rubin et al., 1987).

Before conducting the regression analyses, we assessed the distribution of residuals of the dependent variable(s). In the regression analyses, we used the bootstrap method if the distribution of the residuals was not normal. We used t-tests or Mann-Whitney tests to compare scores for ratings of 'usefulness', for whether participants would recommend the intervention, and the amount of previous experience with yoga, Tai Chi or any type of meditation. All data were analysed using SPSS V25, with the level of significance set at $p<0.05$, except the multiple imputation, which was conducted using Stata V12.

\section{Results}

\section{Baseline characteristics}

\section{Recruitment and exclusions}

Recruitment took place over 2 years from January 2015 to January 2017. As shown in Fig. 1, 220 patients were invited to participate and 73 were excluded. A total of 147 were randomised and 71 of these completed all the followups and were included in the final analysis (see Fig. 1). Recruitment was predominantly from the hospital physiotherapy department $(\mathrm{n}=113)$, with some patients also from pain clinics $(n=34)$. Completeness of follow-ups was similar in the two groups.

Among the 76 'non-completers', a small portion (13\%) reported being too unwell to continue, $23 \%$ reported that they had completed the study but failed to return their study forms and $25 \%$ gave various reasons (e.g., work/family issues). A further $36 \%$ were un-contactable after baseline measures but overall, the dropout rate did not differ between groups (i.e., those randomised to the intervention group and those to the control group).

Baseline demographics and pain characteristics according to study group are presented in Table 2 . The sample as a whole had a mean age of 54 years, over two-thirds were female, close to half were Caucasian, just over half were employed, nearly half were married or living with a partner, and over half had a diagnosis that included back pain.

At baseline the two groups were very similar for all measures (see Tables 2 and 3), except for duration of pain, which was significantly higher for the control group $(p=0.009)$.

\section{Brief measures before and after intervention}

Using adjusted multiple linear regression there were no significant associations between study group and any of the three brief post-intervention scores (Table 4).

\section{Outcomes after 1 month}

After 1 month, we found no significant associations between study group and any change scores (Table 5) with the notable exception of the MBI group having a higher confidence in managing pain compared with the control group (adjusted mean difference of change scores, $\beta=-0.24,95 \% \mathrm{CI},-0.04,1.46$ ).

Results for the individual domains of the EQ-5D-5L are reported instead of an overall patient health state which can be calculated (Devlin et al., 2017) using this instrument, as the domains individually (e.g., pain domain) were of more interest.

Change scores for the CAMS-R were measured at 1 week as well as 1 month but were not significantly associated with study group at either time point.

\section{Acceptability and previous experience outcomes}

Participant ratings of likelihood of recommending the audio and previous experience of activities similar to the audio were not significantly different between groups at 1 week (Table 6). However, the rating of how useful the audio was for relaxing was significantly higher in the MBI group.

As the duration of pain was significantly higher for the control group at baseline, all the regression analyses were repeated adjusting for pain duration at baseline and the results were unchanged.

\section{Missing data}

To address missing data, a sensitivity analysis was conducted using multiple imputation as described in the methods section. Ratings of all measures were analysed and very similar results were produced.

\section{Adherence results}

There were no significant differences between the MBI group at 1 week $(\mathrm{M}=4.58, \mathrm{SD}=1.61)$ and 1 month $(\mathrm{M}=8.50, \mathrm{SD}=4.98)$ when compared to the control group at 1 week $(\mathrm{M}=3.82, \mathrm{SD}=1.24)$ and 1 month $(\mathrm{M}=6.52, \mathrm{SD}=3.22)$ in relation to the number of times patients self-reported listening to the audio. 
Fig. 1 CONSORT flow

diagram of patient participation

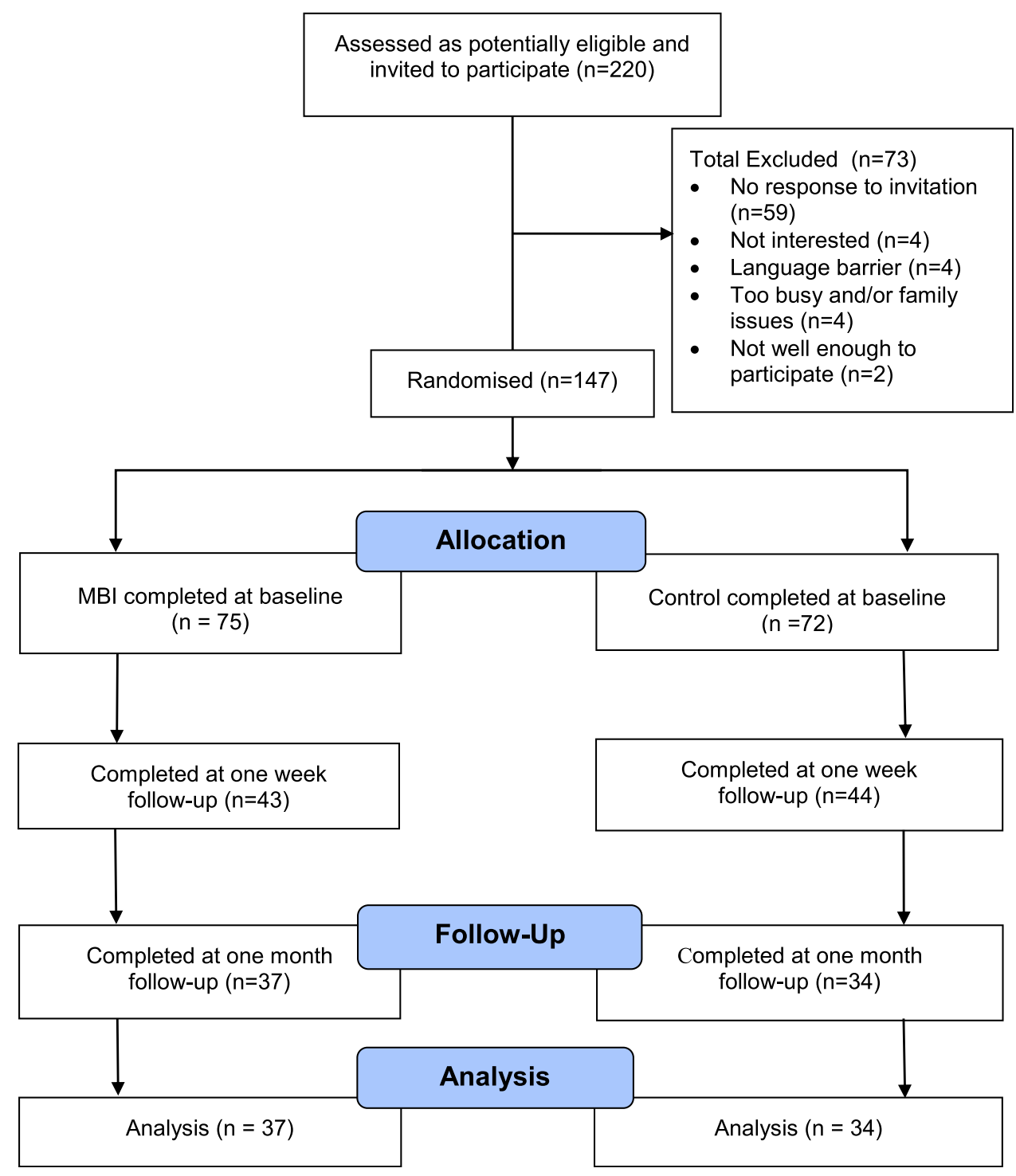

\section{Qualitative analysis of telephone follow-ups at 1 week and 1 month}

Participants were followed up by telephone at 1 week and 1 month for brief interviews. Hand notes were taken and an elementary thematic analysis was conducted for each group separately.

The main theme that emerged at 1 week for the MBI group was about 'how the audio was helpful but it did not take the pain away'. 'Benefits of the audio' was another theme that emerged and it had two sub-themes: 'feeling relaxed' and 'better at coping'. For the control group, 'benefits of the audio' was also a strong theme with two sub-themes of: 'being distracted from the pain' and 'relaxation'. A further theme of 'not feeling much different' overall, also emerged as a secondary theme.

At 1 month, the feedback from the MBI group produced a theme around benefits of the audio again but with three sub-themes this time: 'being a good distraction', 'enhancing coping abilities' and 'better sleep'. One participant qualified this further by reporting that they took substantially less sleep medication since using the MBI. Another two themes emerged which were the 'ease of use' and 'openness to more mindfulness options.' The control group also had a theme of audio benefits again but with the subthemes of 'being distracted' and 'better sleep' this time. As well, the theme about the 'audio not making much a difference' emerged again for this group which is distinct from the MBI group. However, themes about 'ease of use' and the 'audio being an enjoyable experience' emerged for the control group as well.

Finally, when participants in the control group were asked at 1 month if (s)he would like to try the MBI, almost three quarters reported said yes. 
Table 2 Baseline demographic and pain characteristics

\begin{tabular}{|c|c|c|c|}
\hline Variable & MBI $n=37$ No $(\%)$ & Control $\mathrm{n}=34$ No $(\%)$ & Statistic $^{\mathrm{a}} p$ values \\
\hline Age, mean (SD), years & $54.7(12.5)$ & $52.8(12.2)$ & $\begin{aligned} t & =-0.66 \\
p & =0.513\end{aligned}$ \\
\hline Years in education, mean (SD) & $\begin{array}{l}13.0(2.3) \\
\text { Median } 13.0\end{array}$ & $\begin{array}{l}13.0(2.9) \\
\text { Median } 13.0\end{array}$ & $\begin{array}{l}U=625.00 \\
p=0.962\end{array}$ \\
\hline Duration of Pain, mean (SD) years & $\begin{array}{l}8.1(12.7) \\
\text { Median } 2.0\end{array}$ & $\begin{array}{l}11.2(10.5) \\
\text { Median } 7.0\end{array}$ & $\begin{array}{l}U=389.50 \\
\boldsymbol{p}=\mathbf{0 . 0 0 9}\end{array}$ \\
\hline Female & $24(65)$ & $21(62)$ & $\begin{array}{l}\chi^{2}=0.73 \\
p=0.786\end{array}$ \\
\hline Caucasian & $19(51)$ & $11(32)$ & $\begin{array}{c}\chi^{2}=2.60 \\
p=0.105\end{array}$ \\
\hline Employed & $20(54)$ & $14(41)$ & $\begin{array}{c}\chi^{2}=1.18 \\
p=0.278\end{array}$ \\
\hline Married/living with partner & $18(49)$ & $16(47)$ & $\begin{array}{c}\chi^{2}=0.18 \\
p=0.893\end{array}$ \\
\hline Back pain diagnosis & $22(60)$ & $18(53)$ & $\begin{array}{c}\chi^{2}=0.31 \\
p=0.580\end{array}$ \\
\hline Currently receiving pain treatment & $32(87)$ & $29(85)$ & $\begin{array}{c}\chi^{2}=0.02 \\
p=0.885\end{array}$ \\
\hline
\end{tabular}

${ }^{a}$ Chi squared, $t$ test or Mann-Whitney

Significant $p$ values in bold

\section{Discussion}

This study examined the effects of a brief MBI on patients with persistent pain and assessed the feasibility of conducting a definitive RCT. In the adjusted model, compared with the control condition, the MBI did not significantly affect ratings of pain related symptoms, distress or distraction made immediately after the intervention. Results from standardized questionnaires measuring anxiety, depression, mindfulness and quality of life also showed no significant differences between groups at the 1 month follow-up, or at 1 week for mindfulness. At 1 week, ratings for how useful the audio was for relaxation were significantly higher for the MBI group versus the control group. Additionally, at 1 month, with adjustments, ratings of confidence in managing pain were significantly higher for the MBI than the control. Retention was an issue that would need to be addressed prior to a definitive trial, with only around half of those randomised completing the 1 month follow-up.

This study has some notable strengths. First, participants were recruited in clinical settings with a diagnosis of persistent pain, making the findings applicable to patients who would be likely to be offered this intervention in the UK NHS. We are only aware of one previous study that has used a brief MBI with a persistent pain population (Ussher et al., 2012). Secondly, based on prior qualitative research (Howarth et al., 2016a, b), the MBI was specifically developed to target those with persistent pain and does not need to be delivered by a trained specialist. Furthermore, the protocol has been published (Howarth et al., 2016a, b) and the CONSORT checklist and flow diagram were used to guide study design and implementation. To maximize fidelity, researcher checklists and scripts were used to standardize procedures and all groups received an intervention delivered by audio with written instructions to guide use at home. The intervention and control audios were matched for time, pacing and voice. A final strength was the use of a broad range of measures, including some that had been previously shown to be sensitive to the effects of a brief MBI in a pain population.

There were also limitations. There was a high dropout rate, with only around half of those randomised completing all follow-ups. The analysis found that the characteristics of those who dropped out were very similar to those who 'completed' and the results were unchanged when missing data was imputed but it is possible that those who dropped out did not find the intervention acceptable. On reflection, it is possible that the high dropout rate is related to the nature of the population and the care pathway. Recruitment was mostly from physiotherapy clinics and care pathways for pain management within the NHS tend to start with manual therapies (e.g., physiotherapy) and as these, or pharmaceutical treatments, fail to be effective, multicomponent interventions are gradually introduced, usually including psychological components. Thus, it is possible 
Table 3 Outcomes for measures taken at baseline and at 1 month

\begin{tabular}{|c|c|c|c|c|}
\hline \multirow[b]{2}{*}{ Variable $^{\mathrm{a}}$} & \multicolumn{2}{|l|}{ Baseline } & \multicolumn{2}{|l|}{1 month } \\
\hline & $\begin{array}{l}\text { MBI } n=37 \text { Mean } \\
(\mathrm{SD})\end{array}$ & $\begin{array}{l}\text { Control } n=34 \text { Mean } \\
(\mathrm{SD})\end{array}$ & $\begin{array}{l}\text { MBI } n=37 \text { Mean } \\
(\text { SD) }\end{array}$ & $\begin{array}{l}\text { Control } \mathrm{n}=34 \text { Mean } \\
(\mathrm{SD})\end{array}$ \\
\hline BPI pain severity score $(0-10)$ & $5.6(2.2)$ & $5.4(2.0)$ & $5.8(2.2)$ & $5.4(2.0)$ \\
\hline BPI pain interference score $(0-10)$ & $5.3(2.4)$ & $6.0(2.9)$ & $5.3(2.4)$ & $5.6(2.9)$ \\
\hline BPI Overall score $(0-10)$ & $5.5(2.2)$ & $5.5(2.3)$ & $5.5(2.1)$ & $5.5(2.3)$ \\
\hline CAMS-R mindfulness score $(0-40)$ & $27.1(7.3)$ & $24.7(6.8)$ & $26.7(5.6)$ & $26.6(7.8)$ \\
\hline \multirow[t]{2}{*}{ EQ-5D level: mobility (1-5) } & $2.3(1.0)$ & $2.4(1.3)$ & $2.2(0.9)$ & $2.4(1.3)$ \\
\hline & Median 2.0 & Median 2.0 & Median 2.0 & Median 2.0 \\
\hline \multirow[t]{2}{*}{ EQ-5D level: self-care $(1-5)$} & $1.6(0.8)$ & $1.9(1.1)$ & $1.5(0.9)$ & $2.0(1.1)$ \\
\hline & Median 1.0 & Median 2.0 & Median 2.0 & Median 2.0 \\
\hline \multirow[t]{2}{*}{ EQ-5D level: usual activities (1-5) } & $2.8(0.9)$ & $2.7(1.2)$ & $2.7(0.9)$ & $2.4(1.1)$ \\
\hline & Median 3.0 & Median 3.0 & Median 2.0 & Median 2.0 \\
\hline \multirow[t]{2}{*}{ EQ-5D level: pain and discomfort (1-5) } & $3.4(0.9)$ & $3.2(0.9)$ & $3.1(1.0)$ & $3.2(1.1)$ \\
\hline & Median 3.0 & Median 3.0 & Median 3.0 & Median 3.0 \\
\hline \multirow[t]{2}{*}{ EQ-5D level: anxiety and depression (1-5) } & $2.1(1.0)$ & $2.4(1.2)$ & $2.0(1.1)$ & $2.4(1.3)$ \\
\hline & Median 2.0 & Median 2.0 & Median 2.0 & Median 2.0 \\
\hline \multirow[t]{2}{*}{ EQ-5D VAS $(0-100)$} & $60.0(21.5)$ & $60.6(23.2)$ & $60.8(21.4)$ & $58.8(20.4)$ \\
\hline & Median 60.0 & Median 65.0 & Median 67.0 & Median 60.0 \\
\hline \multirow[t]{2}{*}{ HADS anxiety score $(0-21)$} & $8.4(3.8)$ & $9.9(4.8)$ & $7.6(4.3)$ & $8.7(4.0)$ \\
\hline & Median 9.0 & Median 9.5 & Median 7.0 & Median 8.0 \\
\hline \multirow[t]{2}{*}{ HADS depression score $(0-21)$} & $6.8(3.7)$ & $9.3(7.2)$ & $5.8(3.7)$ & $7.6(5.0)$ \\
\hline & Median 7.0 & Median 8.5 & Median 6.0 & Median 8.0 \\
\hline \multirow[t]{2}{*}{ PCS score $(0-42)$} & $22.2(11.5)$ & $21.7(13.7)$ & $18.3(13.3)$ & $21.0(12.2)$ \\
\hline & Median 25.0 & 20.5 & Median 16.0 & Median 22.0 \\
\hline \multirow{2}{*}{$\begin{array}{l}\text { Level of confidence in managing pain (1-7), } \\
\text { mean (SD) }\end{array}$} & $3.5(1.6)$ & $4.1(1.2)$ & $4.5(1.0)$ & $4.2(1.3)$ \\
\hline & Median 4.0 & Median 4.0 & Median 4.0 & Median 4.0 \\
\hline \multirow[t]{2}{*}{ Level of ADLlimitation (1-7), mean (SD) } & $3.5(1.2)$ & $3.3(1.1)$ & $3.0(0.8)$ & $2.9(1.0)$ \\
\hline & Median 4.0 & Median 3.0 & Median 3.0 & Median 3.0 \\
\hline
\end{tabular}

${ }^{a}$ BPI (Brief Pain Inventory), HADS (Hospital Anxiety and Depression Scale), PCS (Pain Catastrophizing Score), EQ-5d (EuroQoL 5 Dimensions), CAMS-R (Cognitive Awareness and Mindfulness Scale—Revised), ADL (activities of daily living)

Table 4 Adjusted $^{\mathrm{a}}$ associations $^{\mathrm{b}}$ between groups and post-intervention scores for brief measures in clinic and in participants' own environment

\begin{tabular}{|c|c|c|c|}
\hline Outcome variable ${ }^{c}$ & Unstandardized Beta Coefficients $^{\mathrm{d}}$ & Standardized Beta Coefficients ${ }^{\mathrm{d}}(95 \% \mathrm{CI})$ & $p$ \\
\hline \multicolumn{4}{|c|}{ Right now, I could be easily distracted } \\
\hline Clinic & -0.09 & $-0.03(-0.66,0.49)$ & 0.768 \\
\hline Own environment & -0.50 & $-0.17(-1.13,0.13)$ & 0.120 \\
\hline \multicolumn{4}{|c|}{ How severe are your pain related symptoms right now? } \\
\hline Clinic & -0.12 & $-0.04(-0.64,0.40)$ & 0.650 \\
\hline Own environment & -0.31 & $-0.11(-0.80,0.19)$ & 0.225 \\
\hline \multicolumn{4}{|c|}{ How distressing are your pain related symptoms right now? } \\
\hline Clinic & -0.15 & $-0.05(-0.76,0.47)$ & 0.633 \\
\hline Own environment & -0.41 & $-0.13(-0.95,0.14)$ & 0.138 \\
\hline
\end{tabular}

${ }^{a}$ Adjusted for age, sex, Brief Pain Inventory total score, pain duration and pre-intervention score

${ }^{\mathrm{b}}$ Using multiple linear regression

${ }^{\mathrm{c}}$ All items were rated from $1=$ not at all to $7=$ extremely

${ }^{\mathrm{d}}$ The direction of the effect positively favoured the MBI group 
Table 5 Adjusted $^{\mathrm{a}}$ associations $^{\mathrm{b}}$ between study groups and change scores at 1 month

\begin{tabular}{lccc}
\hline Outcome variable $^{\mathrm{c}}$ & Unstandardized beta coefficients $^{\mathrm{d}}$ (95\% CI) & Standardized beta coefficients $^{\mathrm{d}}(95 \%$ CI $)$ & $p$ \\
\hline CAMS-R mindfulness score (0-40) & 2.31 & $0.17(-1.03,5.65)$ & 0.171 \\
EQ-5D level: mobility (1-5) & -0.01 & $-0.01(-0.32,0.29)$ & 0.942 \\
EQ-5D level: self-care (1-5) & 0.24 & $0.19(-0.05,0.52)$ & 0.104 \\
EQ-5D level: usual activities (1-5) & -0.14 & $-0.10(-0.48,0.20)$ & 0.414 \\
EQ-5D level: pain and discomfort (1-5) & 0.32 & $0.20(-0.06,0.69)$ & $0.02(-0.48,0.56)$ \\
EQ-5D level: anxiety and depression (1-5) & 0.04 & $-0.05(-12.42,7.89)$ & 0.887 \\
EQ-5D VAS (0-100) & -2.26 & $-0.08(-2.05,1.07)$ \\
HADS anxiety score (0-21) & -0.50 & $-0.08(-3.32,1.64)$ & 0.658 \\
HADS depression score (0-21) & -0.84 & $0.14(-2.20,7.95)$ \\
PCS score (0-42) & 2.88 & $0.24(0.04,1.46)$ \\
Level of confidence in managing pain (1-7) & 0.75 & $0.11(-0.25,0.73)$ & 0.532 \\
Level of ADL ${ }^{b}$ limitation (1-7) & 0.24 & 0.261 \\
\hline
\end{tabular}

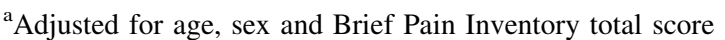

${ }^{\mathrm{b}}$ Using multiple linear regression

${ }^{\mathbf{c}}$ BPI (Brief Pain Inventory), HADS (Hospital Anxiety and Depression Scale), PCS (Pain Catastrophizing Score), EQ-5d (EuroQoL 5 Dimensions), CAMS-R (Cognitive Awareness and Mindfulness Scale-Revised), ADL (activities of daily living)

${ }^{\mathrm{d}}$ The direction of the effect positively favoured the MBI group, except for CAMS-R

Significant $p$ values in bold

Table 6 Ratings for usefulness, recommendation and previous experience at one week and mindfulness after one week

\begin{tabular}{|c|c|c|c|c|}
\hline Secondary outcomes & $\begin{array}{l}\text { MBI } n=37 \\
\text { Mean }(\mathrm{SD})\end{array}$ & $\begin{array}{l}\text { Control } \mathrm{n}=34 \\
\text { Mean }(\mathrm{SD})\end{array}$ & $U^{\mathrm{a}}$ & $p$ \\
\hline $\begin{array}{l}\text { "How useful did you find the audio guide for helping you to relax?" }(1=\text { not at all to } \\
5=\text { extremely useful) }\end{array}$ & $3.5(0.9)$ & $3.1(1.1)$ & $U=461.00$ & 0.044 \\
\hline $\begin{array}{l}\text { "Would you recommend this audio guide to others to help manage their persistent pain?" } \\
(1=\text { definitely would not recommend to } 5=\text { definitely would recommend it })\end{array}$ & $4.1(0.8)$ & $3.8(1.0)$ & $U=476.00$ & 0.062 \\
\hline $\begin{array}{l}\text { "Have you had experience of yoga, tai-chi or any type of meditation?" }(1=\text { no } \\
\text { experience of these activities to } 7=\text { I currently practice these activities at least once a } \\
\text { week) }\end{array}$ & $2.8(1.9)$ & $2.7(1.7)$ & $U=625.00$ & 0.962 \\
\hline
\end{tabular}

${ }^{a}$ Data was skewed therefore Mann-Whitney-tests were used and the u value is reported

Significant $p$ values in bold

that as the patients were likely to be unfamiliar with psychological interventions as this stage of their treatment they found it difficult to engage with the MBI. Equally, as adherence was only self-reported, it is unknown how often the participants truly used audio or if they engaged with other types of formal practice that may have influenced results. Furthermore, we omitted a measure of expectancy, regarding the anticipated effects of the interventions, and this information may have contributed to an interpretation of the high dropout rate as well as allowing us to consider whether expectancy had enhanced the effect of either of the interventions.

The rate of recruitment of $48 \%$ was reasonable for this population and type of intervention (Bawa et al., 2015; Ussher et al., 2012). Those who declined to participate reported a mix of reasons such as language, poor health status, lack of time and/or interest. As data was not avail- able for those not recruited, it is unclear whether those recruited are representative of all the patients that were referred. Nevertheless, comparison of this sample with other data from persistent pain populations used in MBI research (Bawa et al., 2015) suggests this sample is representative of persistent pain patients as a whole. Finally, the evaluation was not blinded as limited resources meant the same researcher delivered the intervention and conducted the assessments. To reduce bias overall, a separate researcher conducted analysis and was blinded to treatment allocation.

This is one of only two studies investigating a brief MBI with persistent pain patients and the lack of evidence for the MBI having positive effects immediately post-intervention in the current study is inconsistent with its predecessor (Ussher et al., 2012). While lack of face-to-face interaction with a clinician can detract from the impact of 
interventions, there was a tendency for ratings in both study groups to change in a positive direction so perhaps the potency of the control condition limited detection of unique MBI effects. In the previous study where significant effects were found, the control condition was an audio of an antiquated natural history text, which had little effect on any of the outcomes (Ussher et al., 2012). The current study used a contemporary history text in an attempt to engage participants of a broad age range and for a longer time period than used in the previous study. Although an active control is often considered a positive design feature, this study appeared to have the same challenge as a previous brief MBI study (Zeidan et al., 2010b) where the control offered similar benefits to the intervention (i.e., significantly improved mood). Due to the nature of the interventions (i.e., listening without interruption to an audio in a comfortable position), it is possible that the experience of the control was both relaxing and temporarily distracting. Anecdotally, participants in both groups reported that being advised to take $15 \mathrm{~min}$ time out for themselves was very enjoyable However, based on evidence from the current study, in clinical practice giving people with persistent pain a brief body scan to use at home with little guidance cannot be recommended over using other types of audio. Further research should consider testing for dose effects in case there is a threshold for benefits unique to MBIs (i.e., those routinely found in in full length MBIs) which becomes apparent or potentially a relationship with pain duration as this study included a population with an average duration over 8 years compared with less than 7 years for its predecessor (Ussher et al., 2012).

Measures taken at 1 month were included so as to help define parameters for a definitive trial and were not powered to detect significant changes but despite this, ratings of confidence in managing pain were significantly higher in the MBI group versus the control at this time. This is encouraging as there is evidence supporting the importance of self-efficacy in self-management of persistent pain (Carnes et al., 2012; Damush et al., 2016; Nicholas et al., 2017; Roditi \& Robinson, 2011), and a measure of selfefficacy could be a primary outcome in a definitive trial. Additionally, those in the MBI group rated the intervention as being significantly more useful for relaxation compared with the control group, which is consistent with the commonly reported mindfulness 'side effect' of relaxation (Chang et al., 2011; Dusek et al., 2006). Although it must be acknowledged that the significant effects for both measures may be artefacts of the high number of statistical tests run, it is noteworthy that the MBI group reported the distinct benefit of enhanced coping after 1 month of use, which is consistent with the finding for improved self-efficacy.
This pilot study observed a lack of immediate effects for the MBI versus control group and an attrition rate that needs to be specifically addressed by using alternate retention strategies. However, a reasonable recruitment rate, and significantly higher ratings of usefulness of the MBI and improvements in self-efficacy ratings for the MBI group versus control, suggests that the intervention was reasonably acceptable. To increase retention rates, the recruitment strategy should be revised and perhaps the option of more than one type of MBI (e.g., mindfulness breathing or moving) audio could be offered from the beginning instead of only after 1 week. As the immediate effects were investigated for a mindfulness body scan audio only, it cannot be assumed that all MBIs would have the same result. Based on a recruitment strategy previously used successfully with NHS persistent pain patients (Critchley et al., 2007), patients who have been wait-listed to receive physiotherapy could be invited. This would allow for those who respond to be more likely to selfidentify as being ready to engage with the intervention. Targeting this population may also allow for a larger pool of patients to be contacted and retained, especially if combined with financial incentives to return study forms and a choice of MBI audio (i.e., body scan, breathing or moving) from the start. A nested qualitative study conducted by an independent researcher could also be included and may increase understanding of adherence issues.

Overall, the findings demonstrate that it is likely feasible, pending very specific modifications to recruitment strategies, to engage patients with persistent pain to a study evaluating a brief MBI and that the intervention is acceptable. As this group is particularly difficult to involve in research due to pain management disparities (Campbell et al., 2012), developing self-management interventions is indeed a challenge. However, the use of digital technology offers much potential (Morton et al., 2017) and despite dropout rates ranging from 2 to $83 \%$ for some internetbased trials (Melville et al., 2010), a recent review of internet interventions specific to persistent pain found small to moderate effects overall (Buhrman et al., 2016). As the average length of these intervention was 9 weeks, further investigation into developing effective brief MBIs delivered digitally could be beneficial to many. There is a distinct lack of non-burdensome solutions, so the intervention presented in this study (i.e., a brief MBI audio loaded onto an MP3 player) may be a valid starting point worth pursuing. However, based on the current study, a definitive trial cannot be recommended. While there are no formal plans for this specific intervention, disseminating the recruitment challenges is probably the most constructive action. It is strongly recommended that future research focus on refined recruitment strategies to target participants who self-identify as ready to engage (e.g., not through 
practitioner referral). This way, retention of participants may be maximized and the potential use of an intervention that takes little time and resources from the perspective of both patient and healthcare provider, may be more fully evaluated.

Acknowledgements This research has been conducted independently by St George's, University of London. We would like to thank all the patients participating in the study for their valuable support as well as the physiotherapists who were kind enough to make the referrals from St John's Therapy Centre and St George's Hospital outpatient physiotherapy service, and the pain consultant, Dr. Oliver Seyfried who made referrals from the Chronic Pain Service at St George's Hospital. The authors would also like to acknowledge Cheryl Watson for her brilliant assistance in trial administration. Much gratitude goes to Breathworks for generously contributing their mindfulness meditation scripts on which the intervention is based and finally, we would like to thank Martha Dancy for providing the voice over narration for every audio used, across this study and its predecessor

\section{Compliance with ethical standards}

Conflict of interest Ana Howarth, Muhammad Riaz, Linda PerkinsPorras, Jared G. Smith, Jeevakan Subramania, Claire Copland, Mike Hurley, Iain Beith, and Michael Ussher declare they have no conflicts of interest.

Human and animal rights and Informed consent All procedures performed in studies involving human participants were in accordance with the ethical standards of the institutional and/or national research committee and with the 1964 Helsinki declaration and its later amendments or comparable ethical standards. This study does not contain any studies with animals performed by any of the authors. Informed consent was obtained from all individual participants included in the study.

Open Access This article is distributed under the terms of the Creative Commons Attribution 4.0 International License (http:// creativecommons.org/licenses/by/4.0/), which permits unrestricted use, distribution, and reproduction in any medium, provided you give appropriate credit to the original author(s) and the source, provide a link to the Creative Commons license, and indicate if changes were made.

\section{References}

Azur, M. J., et al. (2011). Multiple imputation by chained equations: What is it and how does it work? International Journal of Methods in Psychiatric Research, 20, 40-49. https://doi.org/10. 1002/mpr.329

Baer, R. (2003) 'Mindfulness training as a clinical intervention: A conceptual and empirical review', Clinical Psychology: Science and Practice 10:125-143

Baer, R. A., et al. (2006). Using self-report assessment methods to explore facets of mindfulness. Assessment, 13, 27-45. https://doi. org/10.1177/1073191105283504

Bawa, F. L. M., et al. (2015). Does mindfulness improve outcomes in patients with chronic pain? Systematic review and meta-analysis. The British Journal of General Practice: The Journal of the Royal College of General Practitioners, 65, e387-e400. https:// doi.org/10.3399/bjgp15x685297
Bowen, D. J., et al. (2009). How we design feasibility studies. American Journal of Preventive Medicine, 36, 452-457. https:// doi.org/10.1016/j.amepre.2009.02.002

BPS. (2008). The British Pain Society-FAQs. https://www. britishpainsociety.org/people-with-pain/frequently-askedquestions/

Brazier, J. \& Longworth, L. (2011). NICE DSU technical support document 8: An introduction to the measurement and valuation of health for NICE submissions report by the Decision Support Unit.

Bridges, S. (2012). Health Survey for England 2011: Chronic pain (Chapter 9, pp. 291-232).

Breathworks. (2013). Breathworks mindfulness. The Breathworks Foundation. http://www.breathworks-mindfulness.org.uk/

British Pain Society. (2012). National pain audit final report 2010-2012. Dr Foster Intelligence, British Pain Society, Healthcare Quality Improvement Partnership. Retrieved from http://www.nationalpainaudit.org/media/files/NationalPainAudit2012.pdf

Brooks, R. (1996). EuroQol: The current state of play. Health Policy, $37,53-72$.

Brown, K. W., \& Ryan, R. M. (2003). The benefits of being present: Mindfulness and its role in psychological well-being. Journal of Personality and Social Psychology, 84, 822-848.

Buhrman, M., Gordh, T., \& Andersson, G. (2016). Internet interventions for chronic pain including headache: A systematic review. Internet Interventions, 4, 17-34. https://doi.org/10.1016/J. INVENT.2015.12.001

Campbell, L. C., et al. (2012). Challenges and opportunities in pain management disparities research: Implications for clinical practice, advocacy, and policy. The Journal of Pain, 13, 611-619. https://doi.org/10.1016/j.jpain.2012.02.004

Carnes, D., et al. (2012). Effective delivery styles and content for selfmanagement interventions for chronic musculoskeletal pain: A systematic literature review. The Clinical Journal of Pain, 28, 344-354. https://doi.org/10.1097/ajp.0b013e31822ed2f3

Chan, A.-W., et al. (2013). SPIRIT 2013 statement: Defining standard protocol items for clinical trials. American College of Physicians, 158, 200-207. https://doi.org/10.7326/0003-4819-158-3201302050-00583

Chang, B.-H., Dusek, J. A., \& Benson, H. (2011). Psychobiological changes from relaxation response elicitation: Long-term practitioners vs. novices. Psychosomatics, 52, 550-559. https://doi. org/10.1016/j.psym.2011.05.001

Cheung, P. W. H. et al. (2016). Psychometric validation of the EuroQoL 5-Dimension 5-Level (EQ-5D-5L) in Chinese patients with adolescent idiopathic scoliosis. Scoliosis Spinal Disord, 11, 19.

Cleeland, C. S., \& Ryan, K. M. (1994). Pain assessment: Global use of the Brief Pain Inventory. Annals of the Academy of Medicine, Singapore, 23, 129-138.

Crawford, J. R., et al. (2001). Normative data for the HADS from a large non-clinical sample. British Journal of Clinical Psychology, 40, 429-434. https://doi.org/10.1348/014466501163904

Critchley, D. J., et al. (2007). Effectiveness and cost-effectiveness of three types of physiotherapy used to reduce chronic low back pain disability. Spine, 32, 1474-1481. https://doi.org/10.1097/ BRS.0b013e318067dc26

Damush, T. M., et al. (2016). Pain self-management training increases self-efficacy, self-management behaviours and pain and depression outcomes. European Journal of Pain, 20, 1070-1078. https://doi.org/10.1002/ejp.830

Devlin, N. J., et al. (2017). Valuing health-related quality of life: An EQ-5D-5L value set for England. Health Economics., 27, 7. https://doi.org/10.1002/hec.3564 
Dorman, P. J., et al. (1997). Is the EuroQol a valid measure of healthrelated quality of life after stroke? Stroke, 28, 1876-1882. https://doi.org/10.1161/01.STR.28.10.1876

Dusek, J. A., et al. (2006). Association between oxygen consumption and nitric oxide production during the relaxation response. Medical Science Monitor: International Medical Journal of Experimental and Clinical Research, 12, CR1-CR10.

Eccleston, C., Williams, A. C. C., \& Morley, S. (2009). Psychological therapies for the management of chronic pain (excluding headache) in adults. The Cochrane Database of Systematic Reviews, 11, CD007407. https://doi.org/10.1002/14651858. cd007407.pub2

Elliott, T., Renier, C., \& Palcher, J. (2003). Chronic pain, depression, and quality of life: Correlations and predictive value of the SF36. Pain Medicine, 4, 331-339.

EuroQol Group. (1990). EuroQol-A new facility for the measurement of health-related quality of life. Health Policy, 16, 199-208.

Fayaz, A., et al. (2016). Prevalence of chronic pain in the UK: A systematic review and meta-analysis of population studies. $B M J$ Open, 6, e010364. https://doi.org/10.1136/bmjopen-2015-010364

Feldman, G., \& Hayes, A. (2005). Preparing for problems: A measure of mental anticipatory processes. Journal of Research in Personality, 39, 487-516. https://doi.org/10.1016/j.jrp.2004.05. 005

Feldman, G., et al. (2007). Mindfulness and emotion regulation: The development and initial validation of the cognitive and affective mindfulness scale-revised (CAMS-R). Journal of Psychopathology and Behavioral Assessment, 29, 177-190.

Fredheim, O. M. S., et al. (2008). Chronic non-malignant pain patients report as poor health-related quality of life as palliative cancer patients. Acta Anaesthesiologica Scandinavica, 52, 143-148. https://doi.org/10.1111/j.1399-6576.2007.01524.x

Gjeilo, K. H., et al. (2007). Validation of the brief pain inventory in patients six months after cardiac surgery. Journal of Pain and Symptom Management, 34, 648-656. https://doi.org/10.1016/j. jpainsymman.2007.01.010

Goyal, M., et al. (2014). Meditation programs for psychological stress and well-being: A systematic review and meta-analysis. JAMA Internal Medicine, 174, 357-368. https://doi.org/10.1001/ jamainternmed.2013.13018

Greeson, J. M. (2008). Mindfulness research update: 2008. Complementary Health Practice Review, 14, 10-18.

Grossman, P., et al. (2004). Mindfulness-based stress reduction and health benefits: A meta-analysis. Journal of Psychosomatic Research, 57, 35-43. https://doi.org/10.1016/S0022-399 9(03)00573-7

Harrison, A. M., et al. (2017). Are we speaking the same language? Finding theoretical coherence and precision in "mindfulnessbased mechanisms" in chronic pain. Pain Medicine, 18, $2138-2151$.

Hayes, S. C. (2004). Acceptance and commitment therapy, relational frame theory, and the third wave of behavioral and cognitive therapies*. Behavior Therapy, 35, 639-665. https://doi.org/10. 1016/S0005-7894(04)80013-3

Herdman, M., et al. (2011). Development and preliminary testing of the new five-level version of EQ-5D (EQ-5D-5L). Quality of Life Research, 20, 1727-1736. https://doi.org/10.1007/s11136-0119903-x

Hilton, L., et al. (2017). Mindfulness meditation for chronic pain: Systematic review and meta-analysis. Annals of Behavioral Medicine, 51, 199-213. https://doi.org/10.1007/s12160-0169844-2

Hoffmann, T. C., et al. (2014). Better reporting of interventions: Template for intervention description and replication (TIDieR) checklist and guide. BMJ (Clinical research ed.), 348, g1687.
Hofmann, S. G., et al. (2010). The effect of mindfulness-based therapy on anxiety and depression: A meta-analytic review. Journal of Consulting and Clinical Psychology, 78, 169-183. https://doi.org/10.1037/a0018555

Howarth, A., Perkins-Porras, L., Copland, C., et al. (2016a). Views on a brief mindfulness intervention among patients with long-term illness. BMC Psychology, 4, 56. https://doi.org/10.1186/s40359016-0163-y

Howarth, A., Perkins-Porras, L., Smith, J. G., et al. (2016b). Pilot study evaluating a brief mindfulness intervention for those with chronic pain: Study protocol for a randomized controlled trial. Trials, 17, 273. https://doi.org/10.1186/s13063-016-1405-2

Hurst, N. P., et al. (1994). Validity of Euroqol-A generic health status instrument-In patients with rheumatoid arthritis: Economic and Health Outcomes Research Group. British Journal of Rheumatology, 33, 655-662.

Kabat-Zinn, J. (1982). An outpatient program in behavioral medicine for chronic pain patients based on the practice of mindfulness meditation: Theoretical considerations and preliminary results. General Hospital Psychiatry, 4, 33-47.

Kabat-Zinn, J. (1990). Full catastrophe living: Using the wisdom of your body and mind to face stress, pain, and illness. New York: Dell Publishing.

Kabat-Zinn, J., Lipworth, L., \& Burney, R. (1985). The clinical use of mindfulness meditation for the self-regulation of chronic pain. Journal of Behavioral Medicine, 8, 163-190. https://doi.org/10. 1007/BF00845519

Kalia, L. V., \& O'Connor, P. W. (2005). Severity of chronic pain and its relationship to quality of life in multiple sclerosis. Multiple Sclerosis, 11, 322-327. https://doi.org/10.1191/1352458 $505 \mathrm{~ms} 1168 \mathrm{oa}$

Little, R. J. A. (1988). Missing-data adjustments in large surveys. Journal of Business \& Economic Statistics, 6, 287-296. https:// doi.org/10.1080/07350015.1988.10509663

Liu, X., Chang, S., Chen, W., \& Si, M. (2013). Effect of brief mindfulness intervention on tolerance and distress of pain induced by cold-pressor task. Stress Health, 29, 199-204.

Marra, C. A., et al. (2005). A comparison of generic, indirect utility measures (the HUI2, HUI3, SF-6D, and the EQ-5D) and diseasespecific instruments (the RAQoL and the HAQ) in rheumatoid arthritis. Social Science \& Medicine, 60, 1571-1582. https://doi. org/10.1016/j.socscimed.2004.08.034

Melville, K. M., Casey, L. M., \& Kavanagh, D. J. (2010). Dropout from Internet-based treatment for psychological disorders. British Journal of Clinical Psychology, 49, 455-471. https:// doi.org/10.1348/014466509X472138

Michie, S., et al. (2013). The behavior change technique taxonomy (v1) of 93 hierarchically clustered techniques: Building an international consensus for the reporting of behavior change interventions. Annals of Behavioral Medicine, 46, 81-95.

Morley, S., Eccleston, C., \& Williams, A. (1999). Systematic review and meta-analysis of randomized controlled trials of cognitive behaviour therapy and behaviour therapy for chronic pain in adults, excluding headache. Pain, 80, 1-13.

Morris, T. P., White, I. R., \& Royston, P. (2014). Tuning multiple imputation by predictive mean matching and local residual draws. BMC Medical Research Methodology, 14, 75. https://doi. org/10.1186/1471-2288-14-75

Morton, K., et al. (2017). Using digital interventions for selfmanagement of chronic physical health conditions: A metaethnography review of published studies. Patient Education and Counseling, 100, 616-635. https://doi.org/10.1016/J.PEC.2016. 10.019

Mustur, D., et al. (2009). 'Assessment of functional disability and quality of life in patients with ankylosing spondylitis. Srpski arhiv za celokupno lekarstvo, 137, 524-528. 
NCCMH. (2009). Depression: The treatment and management of depression in adults (NICE clinical guideline 90). Retrieved from https://www.nice.org.uk/guidance/cg90

Nicholas, M. K., et al. (2017). Long-term outcomes from training in self-management of chronic pain in an elderly population. Pain, 158, 86-95. https://doi.org/10.1097/j.pain.0000000000000729

Obradovic, M., Lal, A., \& Liedgens, H. (2013). Validity and responsiveness of EuroQol-5 dimension (EQ-5D) versus Short Form-6 dimension (SF-6D) questionnaire in chronic pain. Health and Quality of Life Outcomes, 11, 110. https://doi.org/10.1186/ 1477-7525-11-110

Osman, A., et al. (2000). The pain catastrophizing scale: Further psychometric evaluation with adult samples. Journal of Behavioral Medicine, 23, 351-365. https://doi.org/10.1023/A:1005548801037

Pallant, J. F. \& Bailey, C. M. (2005). Assessment of the structure of the hospital anxiety and depression scale in musculoskeletal patients. Health Quality Life Outcomes, 3, 82.

Prins, B., Decuypere, A., \& Van Damme, S. (2014). Effects of mindfulness and distraction on pain depend upon individual differences in pain catastrophizing: An experimental study. European Journal of Pain, 18, 1307-1315. https://doi.org/10. 1002/j.1532-2149.2014.491.x

Rice, A. S. C., Smith, B. H., \& Blyth, F. M. (2016). Pain and the global burden of disease. Pain, 157, 791-796. https://doi.org/10. 1097/j.pain.0000000000000454

Roditi, D., \& Robinson, M. E. (2011). The role of psychological interventions in the management of patients with chronic pain. Psychology Research and Behavior Management, 4, 41-49. https://doi.org/10.2147/PRBM.S15375

Rogers, A., \& Kennedy, A. (2008). The United Kingdom Expert Patients Programme: Results and implications from a national evaluation. Medical Journal of Australia, 189, S21-S24.

Rosenstiel, A. K., \& Keefe, F. J. (1983). The use of coping strategies in chronic low back pain patients: Relationship to patient characteristics and current adjustment. Pain, 17, 33-44.

Rubin, D. B. (1986). Statistical matching using file concatenation with adjusted weights and multiple imputations. Journal of Business \& Economic Statistics, 4, 87-94. https://doi.org/10. 1080/07350015.1986.10509497

Rubin, D. B. (1987). Multiple imputation for nonresponse in surveys. New York: Wiley.

Sagheer, M. A., Khan, M. F., \& Sharif, S. (2013). Association between chronic low back pain, anxiety and depression in patients at a tertiary care centre. The Journal of the Pakistan Medical Association, 63, 688-690.

Sharpe, L., et al. (2013). A comparison of the effect of mindfulness and relaxation on responses to acute experimental pain. European Journal of Pain, 17, 742-752. https://doi.org/10.1002/j. 1532-2149.2012.00241.x

Sim, J., \& Lewis, M. (2012). The size of a pilot study for a clinical trial should be calculated in relation to considerations of precision and efficiency. Journal of Clinical Epidemiology, 65, 301-308. https://doi.org/10.1016/j.jclinepi.2011.07.011

Sir Liam Donaldson, C. M. O. (2008). 150 years of the annual report of the chief medical officer: On the state of public health 2008 . Department of Health, Richmond House, 79 Whitehall, London SW1A 2NJ, UK.

Song, C.-Y., et al. (2016). Validation of the brief pain inventory in patients with low back pain. Spine. https://doi.org/10.1097/brs. 0000000000001478

Sullivan, M. J. L., Bishop, S. R., \& Pivik, J. (1995). The pain catastrophizing scale: Development and validation. Psychological Assessment, 7, 524-553.
Tang, N. K. Y., Wright, K. J., \& Salkovskis, P. M. (2007). Prevalence and correlates of clinical insomnia co-occurring with chronic back pain. Journal of Sleep Research, 16, 85-95. https://doi.org/ 10.1111/j.1365-2869.2007.00571.x

Thompson, B. L., \& Waltz, J. (2007). Everyday mindfulness and mindfulness meditation: Overlapping constructs or not? Personality and Individual Differences, 43, 1875-1885. https://doi.org/ 10.1016/j.paid.2007.06.017

Urbaniak, G., \& Plous, S. (2013). Research randomizer. Retrieved from https://www.randomizer.org/

Ussher, M., et al. (2012). Immediate effects of a brief mindfulnessbased body scan on patients with chronic pain. Journal of Behavioral Medicine, 37, 127-134. https://doi.org/10.1007/ s10865-012-9466-5

Van Buuren, S., Boshuizen, H. C., \& Knook, D. L. (1999). Multiple imputation of missing blood pressure covariates in survival analysis. Statsitics in Medicine, 18, 681-694.

Veehof, M. M., et al. (2011). Acceptance-based interventions for the treatment of chronic pain: A systematic review and metaanalysis. Pain, 152, 533-542. https://doi.org/10.1016/j.pain. 2010.11.002

Vickers, A. J., et al. (2018). Just-in-time consent: The ethical case for an alternative to traditional informed consent in randomized trials comparing an experimental intervention with usual care. Clinical Trials., 15, 3-8. https://doi.org/10.1177/174077451 7746610

Wainwright, M. (2011). The English Village: History and Traditions (1st ed.). London: Michael O’Mara.

Walach, H., et al. (2006). Measuring mindfulness-The Freiburg Mindfulness Inventory (FMI). Personality and Individual Differences, 40, 1543-1555. https://doi.org/10.1016/j.paid.2005.11. 025

WHO (2003) Adherence to long-term therapies: Evidence for action. Williams, A. C. C., Eccleston, C., \& Morley, S. (2012). Psychological therapies for the management of chronic pain (excluding headache) in adults. The Cochrane Database of Systematic Reviews, 11, CD007407. https://doi.org/10.1002/14651858. cd007407.pub3

Zeidan, F., Gordon, N. S., Merchant, J., \& Goolkasian, P. F. (2010a). The effects of brief mindfulness meditation training on experimentally induced pain. Journal of Pain, 11, 199-209.

Zeidan, F., et al. (2010b). Mindfulness meditation improves cognition: Evidence of brief mental training. Consciousness and cognition, 19, 597-605. https://doi.org/10.1016/j.concog.2010. 03.014

Zeidan, F., et al. (2011). Brain mechanisms supporting the modulation of pain by mindfulness meditation. The Journal of Neuroscience, 31, 5540-5548. https://doi.org/10.1523/jneurosci.5791-10.2011

Zeidan, F., et al. (2012). Mindfulness meditation-related pain relief: Evidence for unique brain mechanisms in the regulation of pain. Neuroscience Letters, 520, 165-173. https://doi.org/10.1016/j. neulet.2012.03.082

Zigmond, A. S., \& Snaith, R. P. (1983). The hospital anxiety and depression scale. Acta Psychiatrica Scandinavica, 67, 361-370.

Publisher's Note Springer Nature remains neutral with regard to jurisdictional claims in published maps and institutional affiliations. 